\title{
Systemic therapy augmented by radiotherapy (STAR) effect for brain metastases in a BRAF-mutated melanoma patient with prolonged survival: a case report
}

\author{
Agostino Cristaudo, Antonio Malorgio, Serena Medoro, Antonio Stefanelli \\ Department of Radiotherapy, Azienda Ospedaliero-Universitaria di Ferrara, Ferrara, Italy
}

Received: September 12, 2020

Revised: December 30, 2020

Accepted: January 4, 2021

\section{Correspondence:}

Agostino Cristaudo

Department of Radiotherapy, Azienda

Ospedaliero-Universitaria di Ferrara,

Via Aldo Moro, 44124 Ferrara, Cona,

Italy

Tel: +39-0532-236096

E-mail: A.cristaudo@ospfe.it

ORCID:

https://orcid.org/0000-0002-9387-1109
Brain metastases are common in stage IV malignant melanoma, carrying a prognosis traditionally regarded as severe, with a median survival of few months. Recently introduced systemic therapies as targeted therapy or immunotherapy have significantly improved the prognosis of metastatic melanoma. The optimal association of radiotherapy to such novel treatments has to be clarified. We report on a 43-year-old woman with 10 brain metastases. Three of them were treated with stereotactic radiosurgery (SRS) with complete response even of the untreated lesions. As the patient was BRAF-mutated, she was started on dabrafenib/trametinib. After 8 months she developed new brain metastases, which again responded to a new treatment with SRS. As after 7 months additional lesions appeared, she was treated with whole brain radiotherapy and was started on nivolumab. Twenty months after the first diagnosis of brain metastases the patient is fit without significant clinical and radiological signs of toxicity.

Keywords: Star effect, Brain metastases, Melanoma, Radiotherapy, Stereotactic radiosurgery, Systemic therapy

\section{Introduction}

Brain metastases are a frequent event in many malignancies, the commonest of which are malignant melanoma, lung and breast cancers [1]. They affect $40 \%-60 \%$ of the patients with stage IV melanoma, having carried a dismal prognosis until recently (median survival being weeks if untreated, and less than 1 year for treated patients) [2].

The traditional treatment for multiple brain metastases has been whole brain radiotherapy (WBRT), reserving surgery, stereotactic radiotherapy (SRT) or stereotactic radiosurgery (SRS) for patients with a limited number of lesions (3 or less) [3]. The appearance of novel systemic therapies such as immune checkpoints inhibitors and molecular targeted therapies has dramatically improved the prognosis of metastatic melanoma patients, even of those with brain metastases $[4,5]$.

In this new scenario it's mandatory to reconsider the role of radiotherapy (RT) and find out the best way to combine it with the aforementioned systemic therapies, aiming at improving the outcomes of these patients and minimizing the potential toxicities of such an association. We report on the case of a young woman with multiple melanoma brain metastases who has been treated with target therapy and several courses of RT and who is doing well 20 months after the first diagnosis of brain metastasis.

\section{Case Report}

The informed consent has been signed by the patient. A 43-yearold Caucasian woman was initially diagnosed in 2010 with skin malignant melanoma of the back (Breslow thickness $0.59 \mathrm{~mm}$,

Copyright (C) 2021 The Korean Society for Radiation Oncology

This is an Open Access article distributed under the terms of the Creative Commons Attribution Non-Commercial License (http://creativecommons.org/licenses/by-nc/4.0/) which permits unrestricted non-commercial use, distribution, and reproduction in any medium, provided the original work is properly cited. 
Clark level III, negative lateral and deep margins, negative sentinel lymph node). The follow-up was negative till February 2019, when she was admitted for symptoms of intracranial hypertension.

A brain magnetic resonance (MR) documented at least 10 supratentorial lesions with the features of melanoma brain metastases (Fig. 1) while a computed tomography (CT) showed secondary lesions in both the kidneys and in the left adrenal. The patient also had a lesion of the neck which was pathologically proved to be a melanoma metastasis, carrying mutation of BRAF V600E.

She underwent SRS of the three main brain lesions respectively in the right and in the left frontal lobes and in the right temporal lobe (10 Gy in single fraction to each lesion), and 5 days later was started on dabrafenib/trametinib. It's been chosen to treat those lesions in order to potentially trigger a synergistic effect with a systemic therapy which has a documented effectiveness also for brain metastases as it crosses the blood-brain-barrier [6].

Actually, a CT scan performed 3 months later (Fig. 2) showed significant response of the treated lesions to the SRT and, very interestingly, a significant reduction in size of the other brain metastases which have not been treated.

The subsequent follow-up was negative for more than 8 months, when a MR documented at least 3 new brain secondarisms (October 2019) (Fig. 3). Two of these lesions (in the right parietal lobe and in the right thalamic area) were treated with SRS (10 Gy in single fraction, December 2019); the systemic therapy with dabrafenib/trametinib has been withheld 5 days before and resumed 5 days after the SRS. After 5 months (May 2020), as a follow-up brain MR showed an increase in number of lesions, dabrafenib/trametinib have been interrupted and the patient underwent WBRT (30 Gy in 12 fractions).

Noticeably, in this time there have been no clinical neither radiological signs of toxicity due to the previous RT treatments, particu-

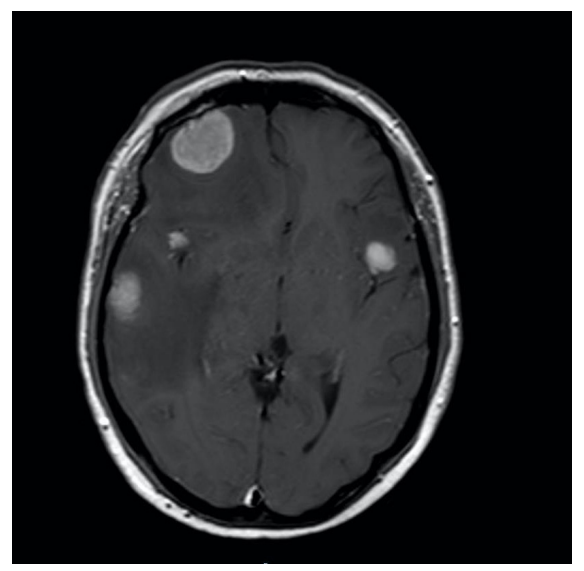

Fig. 1. A brain magnetic resonance image showing at least 10 supratentorial lesions with the features of melanoma brain metastases. larly no signs of radiation necrosis or hemorrhage. In particular, the patient underwent a brain MR in October 2020, showing complete response of the lesions and no new metastases. Subsequently a second line therapy with nivolumab was started. At the moment (October 2020) the patient is fit and well (Karnofsky Performance Score [KPS] of 100).

\section{Discussion}

Malignant melanoma is the third commonest cancer metastasizing to the brain, after lung and breast malignancies [1]. The 40\%-60\% of the patients with stage IV melanoma have brain metastases, and even recent data show a dismal prognosis for those patients (3 to 6 months) [7].

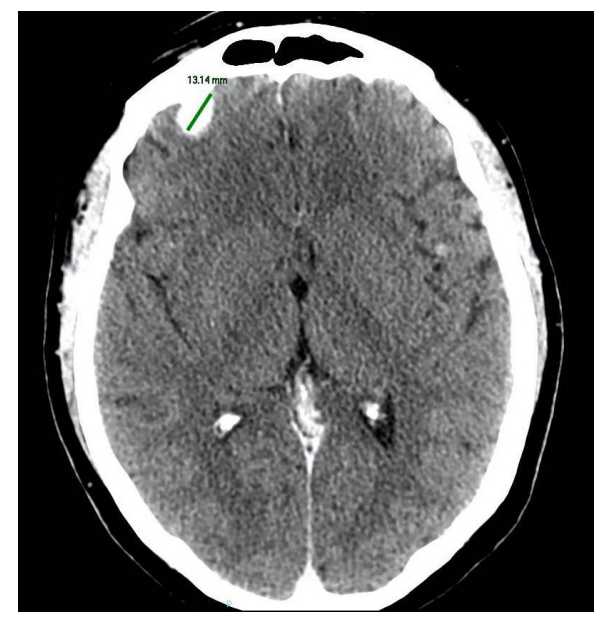

Fig. 2. A computed tomograph scan performed 3 months later showed significant response of the treated lesions to the stereotactic radiotherapy.

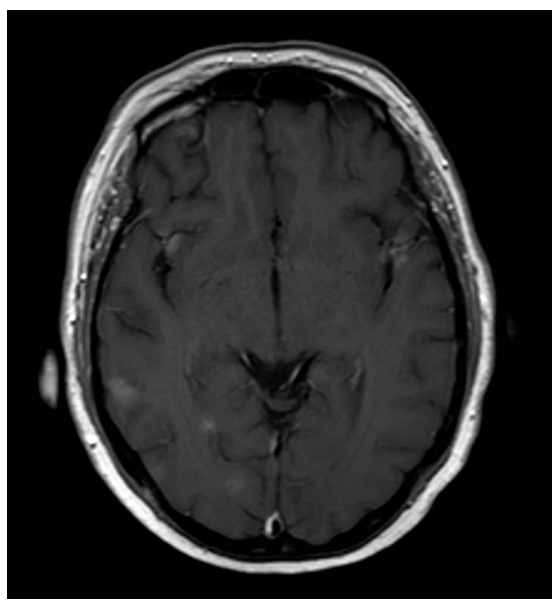

Fig. 3. The subsequent follow-up was negative for more than 8 months, when a magnetic resonance image revealed at least 3 new brain secondarisms (October 2019). 
Even for this reason, such patients have been excluded from clinical trials and traditional treatment for multiple melanoma brain metastases has been WBRT, reserving surgery, SRS or hypofractionated SRT for the cases with a limited number of lesions (3 or less) [3], even if there's an increasing use of SRT also for patients with a greater number of metastases, provided there is a relatively good prognosis and a satisfying performance status [8].

The introduction of novel systemic therapies such as immune checkpoints inhibitors and targeted therapies such as BRAF inhibitor (BRAFi) and mitogen-activated protein kinase inhibitor (MEKi) has dramatically improved the prognosis of stage IV melanoma.

So far, there's shortage of prospective trials regarding the optimal combination of novel systemic therapies and radiotherapy for brain metastases. Nevertheless, nearly all of the current literature agrees that in most cases their association can have promising outcomes being in the same time safe and without significant increase in serious side effects $[1,5,9]$.

Additionally, the use of high doses per fraction makes it possible to take advantage of the abscopal effect, i.e., the phenomenon whereby the irradiation of a lesion causes the regression of other not treated metastases which are distant from the irradiated one $[10,11]$. The proper definition of abscopal effect requires the absence of systemic therapy, so cases in which RT is combined with target therapy and immunotherapy like the present one can be described more precisely as an example of systemic therapy augmented by radiotherapy (STAR) [12]. Consequently it's possible to observe patients with melanoma brain metastases whose overall survival is far longer the traditional historical data.

For instance Saiki et al. [2] have reported on a patient with $B R A F$-mutated melanoma treated with different courses of immunotherapy and vemurafenib and who is fit and well 59 months after treatment with Gamma Knife SRS of 98 brain lesions.

Abu-Gheida et al. [13] described the case of a 45-year-old woman with a BRAF-mutated melanoma with brain metastases. She received WBRT and two Gamma Knife SRS treatments for localized disease progression. After about 6 months she was treated with a second WBRT for ependymal disease progression and subsequently maintained on BRAF and MEK inhibitors with brain disease control for more than a year.

Our case is remarkable first of all because the patient is fit and well without signs of toxicity 20 months after the onset of the first brain lesion; and then because it confirms the possibility of exploiting a potential STAR effect, further improving the treatment's outcome.

Such an outcome, as in the previously mentioned cases, should be considered the result of the synergistic effect of systemic therapies and high-dose hypofractionated RT. In the treatment of brain metastases such systemic therapies must obviously cross the blood-brain-barrier as it's been documented for dabrafenib/trametinib [6].

This case confirms that in patients with melanoma brain metastases it's possible to achieve outcomes which are significantly better than the historical data, so clinicians should always consider an aggressive treatment for these patients, avoiding a nihilistic approach, above all if they have a good performance status.

Radiobiological and translational research, as well as prospective clinical trials, are needed to clarify the optimal association of SRT and systemic therapies such as immunotherapy and target therapy in patients with melanoma brain metastases, with particular consideration of timing and toxicities.

\section{Conflict of Interest}

No potential conflict of interest relevant to this article was reported.

\section{References}

1. van Opijnen MP, Dirven L, Coremans IE, Taphoorn MJ, Kapiteijn $\mathrm{EH}$. The impact of current treatment modalities on the outcomes of patients with melanoma brain metastases: a systematic review. Int J Cancer 2020;146:1479-89.

2. Saiki R, Brill A, Breeze RE. Four-year survival (and counting) after stereotactic radiosurgery to nearly 100 brain metastases: a case report. Cureus 2018;10:e2103.

3. Ali MA, Hirshman BR, Wilson B, et al. Survival patterns of 5750 stereotactic radiosurgery-treated patients with brain metastasis as a function of the number of lesions. World Neurosurg 2017; 107:944-951, e1.

4. Anker CJ, Grossmann KF, Atkins MB, Suneja G, Tarhini AA, Kirkwood JM. Avoiding severe toxicity from combined BRAF inhibitor and radiation treatment: consensus guidelines from the Eastern Cooperative Oncology Group (ECOG). Int J Radiat Oncol Biol Phys 2016;95:632-46.

5. Weaver BD, Goodman JR, Jensen R. Concurrent radiosurgery and systemic therapies for melanoma brain metastases: a systematic review. Cureus. 2019;11:e6147.

6. Davies MA, Saiag P, Robert C, et al. Dabrafenib plus trametinib in patients with BRAFV600-mutant melanoma brain metastases (COMBI-MB): a multicentre, multicohort, open-label, phase 2 trial. Lancet Oncol 2017;18:863-73.

7. Wolf $A$, Zia $S$, Verma $R$, et al. Impact on overall survival of the combination of BRAF inhibitors and stereotactic radiosurgery in patients with melanoma brain metastases. J Neurooncol 2016; 


\section{7:607-15.}

8. Yamamoto $M$, Serizawa $T$, Higuchi $Y$, et al. A multi-institutional prospective observational study of stereotactic radiosurgery for patients with multiple brain metastases (JLGK0901 Study Update): irradiation-related complications and long-term maintenance of mini-mental state examination scores. Int J Radiat Oncol Biol Phys 2017;99:31-40.

9. Trino E, Mantovani C, Badellino S, Ricardi U, Filippi AR. Radiosurgery/stereotactic radiotherapy in combination with immunotherapy and targeted agents for melanoma brain metastases. Expert Rev Anticancer Ther 2017;17:347-56.

10. Petrelli F, De Stefani A, Trevisan F, et al. Combination of radiothera- py and immunotherapy for brain metastases: a systematic review and meta-analysis. Crit Rev Oncol Hematol 2019;144:102830.

11. D'Andrea MA, Reddy GK. Extracranial systemic antitumor response through the abscopal effect induced by brain radiation in a patient with metastatic melanoma. Radiat Oncol J 2019;37: 302-8.

12. Torok JA, Salama JK. Combining immunotherapy and radiotherapy for the STAR treatment. Nat Rev Clin Oncol 2019;16:666-7.

13. Abu-Gheida I, Chao S, Murphy E, et al. Targeted therapy after brain radiotherapy for BRAF-mutated melanoma with extensive ependymal disease with prolonged survival: case report and review of the literature. Front Oncol 2019;9:168. 